PROCEEDINGS OF THE

AMERICAN MATHEMATICAL SOCIETY

Volume 130, Number 1, Pages 167-173

S 0002-9939(01)06096-8

Article electronically published on May 2, 2001

\title{
A SPHERE THEOREM FOR ODD-DIMENSIONAL SUBMANIFOLDS OF SPHERES
}

\author{
THEODOROS VLACHOS
}

(Communicated by Wolfgang Ziller)

\begin{abstract}
We establish a topological sphere theorem from the point of view of submanifold geometry for odd-dimensional submanifolds $M^{n}$ of a unit sphere. We give examples which show that our result is optimal. Moreover, we note the assumption that the dimension $n$ is odd is essential.
\end{abstract}

\section{INTRODUCTION}

One of the most fascinating problems in Riemannian geometry is to find out to what extent several restrictions on curvatures of a compact Riemannian manifold $M$ yield information on the topology of $M$. The classical sphere theorem states that every compact simply connected $n$-dimensional Riemannian manifold with sectional curvatures $K$ satisfying $1<K \leq 4$ is homeomorphic to the $n$-sphere $S^{n}$. The same type of question can be raised for submanifolds of a Riemannian manifold. More precisely, it should be interesting to know how the topology of a submanifold of a Riemannian manifold is affected by conditions on the main intrinsic and extrinsic curvature invariants.

The aim of this note is to establish an optimal sphere theorem from the viewpoint of submanifold geometry for odd-dimensional submanifolds of a unit sphere, in terms of the Ricci curvature and the mean curvature. The proof of our result relies on the Lawson-Simons formula for the nonexistence of stable $k$-currents, which enables us to eliminate the homology groups. We prove the following.

Theorem. Let $M^{n}$ be a compact, oriented n-dimensional submanifold of the unit $(n+k)$-sphere $S^{n+k}$ with mean curvature vector $H$. Assume that the Ricci curvature satisfies

(*) $\quad$ Ric $>\frac{n(n-3)}{n-1}+\frac{n^{2}(n-3)}{(n-1)^{2}}|H|^{2}+\frac{n(n-3)}{(n-1)^{2}}|H| \sqrt{n^{2}|H|^{2}+n^{2}-1}$.

If $n$ is odd and $n>3$, then $M^{n}$ is homeomorphic to $S^{n}$. If $n=3$, then $M^{n}$ is diffeomorphic to a space form of positive sectional curvature.

The class of submanifolds in a sphere which satisfies $(*)$ is not empty. In fact, do Carmo and Warner [1] associated to each positive integer $s$ an isometric minimal immersion of the 3 -sphere $S_{k(s)}^{3}$, with constant sectional curvature $k(s)=$

Received by the editors March 1, 2000 and, in revised form, May 17, 2000.

2000 Mathematics Subject Classification. Primary 53C40; Secondary 53C20.

Key words and phrases. Ricci curvature, mean curvature vector, homology groups.

(C)2001 American Mathematical Society 
$3 / s(s+2) r^{2}$, into the sphere $S^{m(s)}(r)$ of radius $r$, where $m(s)=s(s+2)$. Viewing $S^{m(s)}(r)$ as a hypersphere in the unit sphere $S^{m(s)+1}$ for $r<1$, we get an isometric immersion $\psi_{s}: S_{k(s)}^{3} \rightarrow S^{m(s)+1}$ which satisfies $(*)$. Moreover, Wallach [7] constructed a minimal immersion of an $n$-dimensional complex projective space $P^{n}$ of constant holomorphic curvature $2 n /(n+1) r^{2}$ into the sphere $S^{n(n+2)-1}(r)$ of radius $r$. As in the preceding example, we get an isometric immersion $\phi_{n}: P^{n} \rightarrow S^{n(n+2)}$ with mean curvature $|H|=\sqrt{1-r^{2}} / r$ which satisfies $(*)$ if $n=2$ and $r$ is sufficiently close to 1 . However, $P^{2}$ is not homeomorphic to $S^{4}$. In addition, the standard immersion of $S^{m}(r) \times S^{m}\left(\sqrt{1-r^{2}}\right)$ into $S^{2 m+1}$ satisfies $(*)$ if $r$ is sufficiently close to $\sqrt{1 / 2}$. Nevertheless, $S^{m}(r) \times S^{m}\left(\sqrt{1-r^{2}}\right)$ is not homeomorphic to $S^{2 m}$. These examples justify the necessity of the assumption of the Theorem that $n$ is odd.

On the other hand, we can easily verify that the standard immersion of $S^{m-1}(r) \times$ $S^{m}\left(\sqrt{1-r^{2}}\right)$ into $S^{2 m}$ satisfies

$$
\operatorname{Ric} \geq \frac{n(n-3)}{n-1}+\frac{n^{2}(n-3)}{(n-1)^{2}}|H|^{2}+\frac{n(n-3)}{(n-1)^{2}}|H| \sqrt{n^{2}|H|^{2}+n^{2}-1},
$$

for $r$ sufficiently close to $\sqrt{(m-1) /(2 m-1)}$. This shows that the assumption $(*)$ in the Theorem is optimal. In fact, this example served as the motivation for the choice of the operative condition $(*)$.

We note that a topological sphere theorem from the point of view of submanifold geometry was recently obtained by Shiohama and Xu [5]. They proved the following: Let $M^{n}$ be a complete, oriented $n$-dimensional submanifold of a unit sphere. Assume that the squared length $S$ of the second fundamental form and the mean curvature vector $H$ satisfy $\sup (S-a(n,|H|))<0$, where

$$
a(n,|H|)=n+\frac{n^{3}}{2(n-1)}|H|^{2}-\frac{n(n-2)}{2(n-1)}|H| \sqrt{n^{2}|H|^{2}+4(n-1)} .
$$

Then $M^{n}$ is homeomorphic to $S^{n}$ if $n>3$ or diffeomorphic to a space form of positive sectional curvature if $n=3$.

It is worth noting that there exist submanifolds homeomorphic to a sphere which satisfy $(*)$ and $\sup (S-a(n,|H|))>0$. In fact, the squared length $S$ of the second fundamental form of $\psi_{s}, s \geq 2$, is given by

$$
S=6+\frac{9\left(1-r^{2}\right)}{r^{2}}-\frac{18}{s(s+2) r^{2}}
$$

and

$$
a(n,|H|)=3+\frac{27\left(1-r^{2}\right)}{4 r^{2}}-\frac{3 \sqrt{1-r^{2}}}{4 r^{2}} \sqrt{9-r^{2}} .
$$

Then it is easy to see that $S>a(n,|H|)$, if $r$ is sufficiently close to 1 . This shows that our Theorem does not arise from the result due to Shiohama and $\mathrm{Xu}$ [5].

Remark 1. Our result was proved in [3] under the additional assumption that the submanifold $M^{n}$ is minimal.

\section{Preliminaries}

Let $M^{n}$ be an $n$-dimensional, oriented submanifold of codimension $k$ of the unit $(n+k)$-dimensional sphere $S^{n+k}$ equipped with the induced Riemannian metric $\langle$,$\rangle . Denote the standard connection of S^{n+k}$ by $\bar{\nabla}$, the Riemannian connection of 
$M^{n}$ by $\nabla$, and the second fundamental form by $B$. For tangent vectors $X$ and $Y$ of $M^{n}$, we have the Gauss formula

$$
\bar{\nabla}_{X} Y=\nabla_{X} Y+B(X, Y)
$$

and the Weingarten formula

$$
\bar{\nabla}_{X} e=-A_{e} X+D_{X} e,
$$

where the $(1,1)$ tensor field $A_{e}$ is the shape operator associated with a normal vector field $e$, and $D$ is the connection in the normal bundle of $M^{n}$. It is well known that $\left\langle A_{e} X, Y\right\rangle=\langle B(X, Y), e\rangle$. Let $\left\{e_{1}, \ldots, e_{n}\right\}$ be a local orthonormal frame field in the tangent bundle of $M^{n}$. The mean curvature vector $H$ is given by

$$
H=\frac{1}{n} \sum_{i=1}^{n} B\left(e_{i}, e_{i}\right)
$$

or equivalently

$$
H=\frac{1}{n} \sum_{\alpha=n+1}^{n+k}\left(\operatorname{tr} A_{\alpha}\right) e_{\alpha},
$$

where $\left\{e_{n+1}, \ldots, e_{n+k}\right\}$ is a local orthonormal frame field in the normal bundle of $M^{n}$ and $A_{\alpha}$ denotes the shape operator associated with $e_{\alpha}$. For any unit tangent vector $X$ of $M^{n}$, the Ricci curvature $\operatorname{Ric}(X)$ in the direction of $X$ is given by

$$
\operatorname{Ric}(X)=n-1+\sum_{\alpha=n+1}^{n+k}\left(\operatorname{tr} A_{\alpha}\right)\left\langle A_{\alpha} X, X\right\rangle-\sum_{\alpha=n+1}^{n+k}\left|A_{\alpha} X\right|^{2} .
$$

The main idea in the proof of the Theorem is to show that $M^{n}$ is a homology sphere based on the following result due to Lawson and Simons [4].

Theorem 2.1. Let $M^{n}$ be a compact n-dimensional submanifold of the unit sphere $S^{n+k}$ with second fundamental form $B$, and let $p, q$ be positive integers such that $p+q=n$. If for any point $P \in M^{n}$ and any orthonormal basis $\left\{e_{1}, \ldots, e_{p}, \ldots, e_{n}\right\}$ of the tangent space $T_{P} M$, the inequality

$$
\sum_{i=1}^{p} \sum_{j=p+1}^{n}\left(2\left|B\left(e_{i}, e_{j}\right)\right|^{2}-\left\langle B\left(e_{i}, e_{i}\right), B\left(e_{j}, e_{j}\right)\right\rangle\right)<p q
$$

is satisfied, then $H_{p}\left(M^{n} ; \mathbf{Z}\right)=H_{q}\left(M^{n} ; \mathbf{Z}\right)=0$, where $H_{i}\left(M^{n} ; \mathbf{Z}\right)$ denotes the $i$-th homology group of $M^{n}$ with integer coefficients.

We also need the following auxiliary lemma.

Lemma 2.2. Let $M^{n}$ be an n-dimensional submanifold of $S^{n+k}$ with mean curvature vector $H$. If the Ricci curvature satisfies

$$
\text { Ric }>\frac{n(n-3)}{n-1}+\frac{n^{2}(n-3)}{(n-1)^{2}}|H|^{2}+\frac{n(n-3)}{(n-1)^{2}}|H| \sqrt{n^{2}|H|^{2}+n^{2}-1},
$$

then $n^{2}(n-5)|H|^{2}<4(n+1)$.

Proof. For $n \leq 5$, we have nothing to prove. Hereafter we assume that $n \geq 6$. Our assumption implies that the scalar curvature $\tau$ satisfies

$$
\tau>\frac{n^{2}(n-3)}{n-1}+\frac{n^{3}(n-3)}{(n-1)^{2}}|H|^{2}+\frac{n^{2}(n-3)}{(n-1)^{2}}|H| \sqrt{n^{2}|H|^{2}+n^{2}-1} .
$$


From the Gauss equation, we get $\tau \leq n^{2}|H|^{2}+n(n-1)$. Then we have

$$
(n+1)(n-1)+n(n+1)|H|^{2}>n(n-3)|H| \sqrt{n^{2}|H|^{2}+n^{2}-1},
$$

or equivalently, after squaring and neglecting common factors

$$
n^{2}\left(n^{2}-4 n-1\right)|H|^{4}+n(n+1)\left(n^{2}-5 n+2\right)|H|^{2}-(n+1)^{2}<0 .
$$

From this we obtain, after simplification of the radical term of this quadratic inequality,

$$
|H|^{2}<\frac{n+1}{2 n\left(n^{2}-4 n-1\right)}\left(-n^{2}+5 n-2+(n-3) \sqrt{n(n-4)}\right) .
$$

Now the desired inequality will follow from the above if we prove

$$
\frac{n+1}{2 n\left(n^{2}-4 n-1\right)}\left(-n^{2}+5 n-2+(n-3) \sqrt{n(n-4)}\right)<\frac{4(n+1)}{n^{2}(n-5)} .
$$

Since $\sqrt{n(n-4)}<n-2$ for $n \geq 4$ and $2\left(n^{2}-4 n-1\right)>n(n-5)$ for $n \geq 8$, the previous inequality holds for $n \geq 8$; this inequality is obvious for $n=6$ and 7 .

\section{Proof of the Theorem}

If $n=3$, then it follows by a result due to Hamilton [2] that $M^{n}$ is diffeomorphic to a space form of positive sectional curvature. Hereafter we assume that $n>$ 3 and take any positive integers $p, q$ such that $p+q=n$. Let $P \in M^{n}$ and $\left\{e_{1}, \ldots, e_{p}, \ldots, e_{n}\right\}$ be an arbitrary orthonormal basis of the tangent space $T_{P} M$. We also choose an orthonormal basis $\left\{e_{n+1}, \ldots, e_{n+k}\right\}$ of the normal space at $P$ such that $H=|H| e_{n+1}$ at $P$. Denote by $A_{n+1}, \ldots, A_{n+k}$ the corresponding shape operators. Then we have

$$
\begin{gathered}
\sum_{i=1}^{p} \sum_{j=p+1}^{n}\left(2\left|B\left(e_{i}, e_{j}\right)\right|^{2}-\left\langle B\left(e_{i}, e_{i}\right), B\left(e_{j}, e_{j}\right)\right\rangle\right) \\
=2 \sum_{i=1}^{p} \sum_{j=p+1}^{n} \sum_{\alpha=n+1}^{n+k}\left\langle A_{\alpha} e_{i}, e_{j}\right\rangle^{2}-n|H| \sum_{i=1}^{p}\left\langle A_{n+1} e_{i}, e_{i}\right\rangle+\sum_{\alpha=n+1}^{n+k}\left(\sum_{i=1}^{p}\left\langle A_{\alpha} e_{i}, e_{i}\right\rangle\right)^{2} .
\end{gathered}
$$

By Cauchy-Schwarz inequality we obtain

$$
\begin{gathered}
\sum_{i=1}^{p} \sum_{j=p+1}^{n}\left(2\left|B\left(e_{i}, e_{j}\right)\right|^{2}-\left\langle B\left(e_{i}, e_{i}\right), B\left(e_{j}, e_{j}\right)\right\rangle\right) \\
\leq 2 \sum_{i=1}^{p} \sum_{j=p+1}^{n} \sum_{\alpha=n+1}^{n+k}\left\langle A_{\alpha} e_{i}, e_{j}\right\rangle^{2}-n|H| \sum_{i=1}^{p}\left\langle A_{n+1} e_{i}, e_{i}\right\rangle+p \sum_{\alpha=n+1}^{n+k} \sum_{i=1}^{p}\left\langle A_{\alpha} e_{i}, e_{i}\right\rangle^{2} .
\end{gathered}
$$

We suppose that $p \geq 2$. Then we get

$$
\begin{aligned}
& \sum_{i=1}^{p} \sum_{j=p+1}^{n}\left(2\left|B\left(e_{i}, e_{j}\right)\right|^{2}-\left\langle B\left(e_{i}, e_{i}\right), B\left(e_{j}, e_{j}\right)\right\rangle\right) \\
& \leq p \sum_{i=1}^{p} \sum_{\alpha=n+1}^{n+k}\left|A_{\alpha} e_{i}\right|^{2}-n|H| \sum_{i=1}^{p}\left\langle A_{n+1} e_{i}, e_{i}\right\rangle .
\end{aligned}
$$


In view of (2.1), we obtain

$$
\begin{gathered}
\sum_{i=1}^{p} \sum_{j=p+1}^{n}\left(2\left|B\left(e_{i}, e_{j}\right)\right|^{2}-\left\langle B\left(e_{i}, e_{i}\right), B\left(e_{j}, e_{j}\right)\right\rangle\right) \\
\leq p \sum_{i=1}^{p}\left(n-1-\operatorname{Ric}\left(e_{i}\right)\right)+n(p-1)|H| \sum_{i=1}^{p}\left\langle A_{n+1} e_{i}, e_{i}\right\rangle .
\end{gathered}
$$

For convenience we set

$$
b=\frac{n(n-3)}{n-1}+\frac{n^{2}(n-3)}{(n-1)^{2}}|H|^{2}+\frac{n(n-3)}{(n-1)^{2}}|H| \sqrt{n^{2}|H|^{2}+n^{2}-1} .
$$

Our assumption on the Ricci curvature and (2.1) imply that each eigenvalue $\lambda$ of $A_{n+1}$ satisfies $\lambda^{2}-n|H| \lambda+b-n+1<0$. Hence we have

$$
\lambda<\frac{1}{2}\left(n|H|+\frac{1}{n-1}|-n(n-3)| H\left|+2 \sqrt{n^{2}|H|^{2}+n^{2}-1}\right|\right) .
$$

By virtue of Lemma 2.2, we get

$$
\lambda<\frac{1}{n-1}\left(n|H|+\sqrt{n^{2}|H|^{2}+n^{2}-1}\right)
$$

and therefore

$$
\left\langle A_{n+1} X, X\right\rangle<\frac{1}{n-1}\left(n|H|+\sqrt{n^{2}|H|^{2}+n^{2}-1}\right)
$$

for each unit tangent vector $X$. Then using (3.2) and our assumption, (3.1) yields

$$
\begin{gathered}
\sum_{i=1}^{p} \sum_{j=p+1}^{n}\left(2\left|B\left(e_{i}, e_{j}\right)\right|^{2}-\left\langle B\left(e_{i}, e_{i}\right), B\left(e_{j}, e_{j}\right)\right\rangle\right) \\
<p^{2}(n-1-b)+p(p-1) \frac{n|H|}{n-1}\left(n|H|+\sqrt{n^{2}|H|^{2}+n^{2}-1}\right) .
\end{gathered}
$$

It is obvious that $p \neq q$. Without loss of generality we may assume that $p<q$, hence $n \geq 2 p+1$. Then it follows easily that

$$
p(p-1) \leq p^{2} \frac{n-3}{n-1} .
$$

Therefore we obtain

$$
\sum_{i=1}^{p} \sum_{j=p+1}^{n}\left(2\left|B\left(e_{i}, e_{j}\right)\right|^{2}-\left\langle B\left(e_{i}, e_{i}\right), B\left(e_{j}, e_{j}\right)\right\rangle\right)<p^{2} \frac{n+1}{n-1} \leq p q .
$$

From Theorem 2.1, we infer that $H_{p}\left(M^{n} ; \mathbf{Z}\right)=H_{q}\left(M^{n} ; \mathbf{Z}\right)=0$ for all positive integers $p, q$ such that $2 \leq p, q \leq n-2$ and $p+q=n$. Assume now that $p=1$. Then

$$
\sum_{j=2}^{n}\left(2\left|B\left(e_{1}, e_{j}\right)\right|^{2}-\left\langle B\left(e_{1}, e_{1}\right), B\left(e_{j}, e_{j}\right)\right\rangle\right) \leq 2 \sum_{\alpha=n+1}^{n+k}\left|A_{\alpha} e_{1}\right|^{2}-n|H|\left\langle A_{n+1} e_{1}, e_{1}\right\rangle
$$


and appealing to (2.1) we get

$$
\begin{aligned}
& \sum_{j=2}^{n}\left(2\left|B\left(e_{1}, e_{j}\right)\right|^{2}-\left\langle B\left(e_{1}, e_{1}\right), B\left(e_{j}, e_{j}\right)\right\rangle\right) \\
& \quad \leq 2\left(n-1-\operatorname{Ric}\left(e_{1}\right)\right)+n|H|\left\langle A_{n+1} e_{1}, e_{1}\right\rangle
\end{aligned}
$$

From our assumption and (3.2), we obtain

$$
\begin{aligned}
\sum_{j=2}^{n} & \left(2\left|B\left(e_{1}, e_{j}\right)\right|^{2}-\left\langle B\left(e_{1}, e_{1}\right), B\left(e_{j}, e_{j}\right)\right\rangle\right) \\
& <\frac{2(n+1)}{n-1}+\frac{n^{2}(5-n)}{(n-1)^{2}}|H|^{2}+\frac{n(5-n)}{(n-1)^{2}}|H| \sqrt{n^{2}|H|^{2}+n^{2}-1} \\
& <n-1 .
\end{aligned}
$$

Appealing again to Theorem 2.1, we deduce that $H_{1}\left(M^{n} ; \mathbf{Z}\right)=H_{n-1}\left(M^{n} ; \mathbf{Z}\right)=$ 0 . So $M^{n}$ is a homology sphere. The above arguments can be applied to the Riemannian universal covering $\tilde{M}^{n}$ of $M^{n}$ since $(*)$ implies that the Ricci curvature is positive, bounded away from zero and $\tilde{M}^{n}$ is compact by Myers' Theorem. Since $\tilde{M}^{n}$ is a homology sphere with fundamental group $\pi_{1}\left(\tilde{M}^{n}\right)=0$, it is also a homotopy sphere. By the generalized Poincaré conjecture (Smale $n \geq 5$, Freedman $n=4$ ) we have that $\tilde{M}^{n}$ is homeomorphic to $S^{n}$. A result due to Sjerve 6] implies that $\pi_{1}\left(M^{n}\right)=0$ and so $M^{n}$ is homeomorphic to $S^{n}$. This completes the proof of the Theorem.

Remark 2. It should be interesting to indicate the maximum homology information given by $(*)$ when $n$ is even. Let $M^{n}$ be a compact, oriented $n$-dimensional submanifold of the unit $(n+k)$-sphere $S^{n+k}$ which satisfies $(*)$ and $n=2 m>4$. Then arguing as in the proof of the Theorem above and appealing to Theorem 2.1, we deduce that $H_{p}\left(M^{n} ; \mathbf{Z}\right)=H_{q}\left(M^{n} ; \mathbf{Z}\right)=0$ for all positive integers $p, q$ such that $p+q=n$ and $p, q \neq m$.

\section{ACKNOWLEDGEMENT}

The author wishes to thank the referee for his close reading of the first draft of this paper which led to various improvements.

\section{REFERENCES}

1. M. P. do Carmo and N. R. Wallach, Minimal immersions of spheres into spheres, Ann. of Math. (2) 93 (1971), 43-62. MR 43:4048

2. R. S. Hamilton, Three-manifolds with positive Ricci curvature, J. Differential Geom. 17 (1982), 255-306. MR 84a:53050

3. Th. Hasanis and Th. Vlachos, Ricci curvature and minimal submanifolds, Pacific J. Math., 197 (2001), 13-24.

4. H. B. Lawson and J. Simons, On stable currents and their application to global problems in real and complex geometry, Ann. of Math. (2) 98 (1973), 427-450. MR 48:2881

5. K. Shiohama and H. Xu, The topological sphere theorem for complete submanifolds, Compositio Math. 107 (1997), 221-232. MR 98i:53080 
6. D. Sjerve, Homology spheres which are covered by spheres, J. London Math. Soc. (2) 6 (1973), 333-336. MR 46:9993

7. N. R. Wallach, Minimal immersions of symmetric spaces into spheres, "Symmetric spaces", Ed. Boothby and Weiss, Dekker, New York, 1972, pp. 1-40. MR 53:11545.

Department of Mathematics, University of Ioannina, Ioannina 45110, Greece

E-mail address: tvlachos@cc.uoi.gr 